\title{
A Link between the Slip Line Density and the Oxidation Resistance of the Fatigue Crack Growth in Alloy 718
}

\author{
H. Ghonem and D. Zheng \\ Mechanics of Materials Laboratory \\ Department of Mechanical Engineering \\ University of Rhode Island \\ Kingston, RI 02881
}

\begin{abstract}
Alloy 718 is a nickel-based superalloy characterized by high creep resistance and good mechanical properties at high temperatures. However, it has been found that oxidation has a great influence of fatigue crack growth behavior of this alloy. The present work focuses on the understanding of the relationship between oxidation mechanisms and the fatigue crack growth behavior. In this attempt, it is argued on the basis of analytical modelling as well as experimental observations that the slip line density in the crack tip region is the key factor in modifying the oxidation resistance of the alloy. Two methods have been proposed in order to examine the validity of this concept. In the first method, the influence of high frequency loading on the subsequent low frequency crack growth behavior in laboratory air environment at $650^{\circ} \mathrm{C}$ has been investigated through the use of a sequential high/low frequency load waveform. The second method relies on locked in global deformation effects. In this, a series of crack growth experiments were carried out on specimens with two levels of prestraining, $1 \%$ and $2 \%$, in addition to specimens with no prior deformation. The parameters that have been examined in these experiments included continuous measurements of crack length and near field crack tip displacements, fractographic analysis of fracture surface facets to identify the corresponding cracking mechanisms, qualitative determination of the slip density in the crack tip region and Auger Spectroscopy analysis for the objective of determining the thickness of surface oxide layers formed during the fracture process.

Results of this work have indicated that prior application of high frequency loading or prestraining of the crack tip region produces a reduction of the subsequent low frequency crack growth rate. This reduction of the crack growth rate has been correlated with the deformationrelated slip line density which was shown to be the rate controlling element of the chromium oxide built-up taking place at the slip line-grain boundary intersection nodes within the crack tip region.
\end{abstract}

\footnotetext{
Superalloys 718, 625,706 and Various Derivatives Edited by E.A. Loria

The Minerals, Metals \& Materials Society, 1994
} 